\title{
NIF wakes up
}

\author{
The first step towards the goal of nuclear fusion triggered by laser beams has been taken with the \\ construction and test firing of the National Ignition Facility in the United States.
}

In the small hours of Thursday 26 February, while most of the residents of California were safely tucked up in bed asleep, one of the largest laser experiments in the world was awakening. Just before 2 a.m., the National Ignition Facility (NIF) in Livermore fired all 192 of its high-power laser beams into a target chamber for the very first time. Each beamline carried a pulse of ultraviolet laser energy with an average of $420 \mathrm{~J}$ into the centre of the target chamber, resulting in a total of $80 \mathrm{~kJ}$ being deposited. From the point of view of energy, this represents twice the 40-kJ capability of the giant OMEGA laser at the University of Rochester, USA, which has been running since 1995 and combines 60 laser beams.

The ultimate goal at NIF is to ramp up the energy of its 192 beams so that a colossal $1.8 \mathrm{MJ}$ of energy can be delivered to a tiny hollow gold target cylinder filled with deuterium and tritium, and thus trigger nuclear fusion. The idea is that the intense nanosecond-duration laser beams generate a 'bath' of soft X-rays that heat the outer layer of the fuel causing it to explode, generating shock waves that will compress its core to 100 times the density of lead. This will cause it to reach a temperature of more than 100 million degrees Celsius and pressures that are thousands of times that of the Earth's atmosphere. The fuel core will then ignite and thermonuclear burn will quickly spread through the compressed fuel, releasing 10 to 100 times more energy than the amount deposited by the laser beams. Well, that's the theory anyway.

The February beam firing marks the unofficial completion of the NIF project, which has taken 10 years to build, cost around US $\$ 3.5$ billion and been plagued by controversy, delays and overspending. The Department of Energy project was originally due for completion by 2004 for a budget of $\$ 1.2$ billion. In 2000 it was bailed out with further funding and an extension of the construction schedule to 2008 , but the project leaders had a tough time persuading members of the US Congress that it should be continued. Finally, the last of the giant project's 6,206 optical components was installed on 26 January this year, ahead of a federal deadline of 31 March for the project's

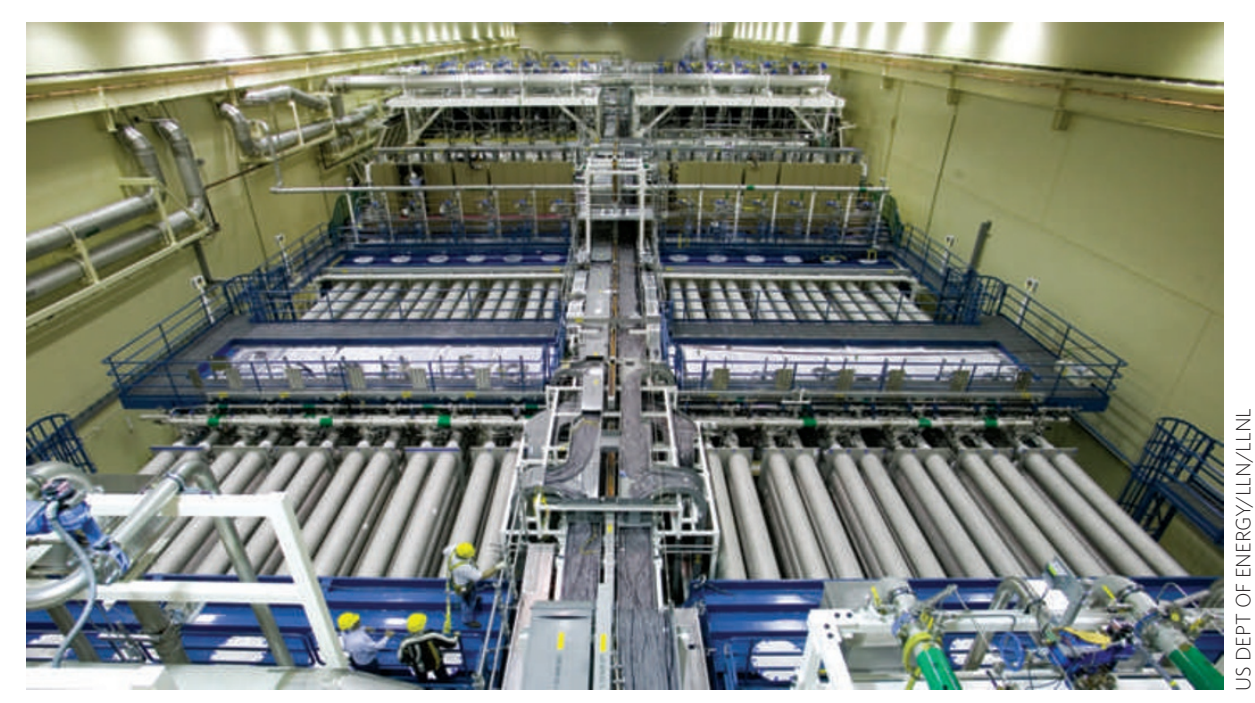

Internal view of one of NIF's laser bays showing several of its beamlines. The facility is now complete.

completion. Although NIF's staff must be breathing a sigh of relief that construction is now finished, the daunting task of achieving fusion and a net energy release is still ahead. The hope is that this will be accomplished within the next two years, with experiments due to commence shortly.

Europe is not far behind in the laser fusion game, with its own project nearing completion. Laser Mégajoule (LMJ), a similar facility to NIF, is being built in Bordeaux, France, by the French atomic agency CEA and is due for completion next year, two years behind schedule. It aims to deliver a similar energy to NIF but will feature 240 beamlines, divided into eight groups of 30 .

LMJ and NIF will be followed by a giant European project called HiPER (the High Power laser Energy Research facility), which was formally launched in October 2008 and is now in a planning and preparatory stage. It is estimated to be operational sometime around 2020. HiPER involves 25 scientific institutions from 11 European nations and the location of the facility has not yet been announced, although the United Kingdom is understood to be a strong contender. Once constructed it hopes to build on the 'proof of principle' results from NIF and LMJ to demonstrate a more commercially practical approach to sustained laser fusion using 'fast ignition', which in principle is more efficient. Unlike NIF and LMJ, which use a single simultaneous burst of energy from all their laser beams both to compress and to heat the target, HiPER will split the process into two discrete stages involving different lasers. One set of beams will compress the target, followed almost instantaneously by a secondary picosecond laser beam that will heat the compressed target and drive it to ignition. The attraction of the scheme is that it relaxes the requirements on the energy of the compression laser beams and the manufacture of the fuel target. The downside is that the scheme is as yet unproven and so there are no guarantees that it will work. Another difference between NIF, LMJ and HiPER is that the latter would be developed as a civilian research facility and would not have the military ties with defence research that have in the past generated criticism and opponents for existing laser fusion schemes. A forerunner test facility to HiPER, called PETAL, is being built in the Aquitaine region in France to test the science behind the fast ignition scheme.

Whether HiPER will provide unequivocal proof that laser fusion is indeed a practical approach to addressing mankind's energy generation problems is not yet clear. What is clear is that much fascinating laser and nuclear physics, a mountain of engineering challenges and a large sum of money will be involved in trying to find out. 